\title{
Stability of an SEIR epidemic model with independent stochastic perturbations
}

\author{
Peter J. Witbooi
}

\begin{abstract}
For an epidemic model of the type mentioned, we prove a theorem on almost sure exponential stability of the disease-free equilibrium. For small values of the diffusion parameter, $\sigma$, we describe the stability of the disease free equilibrium point in terms of an appropriate analogue, $\mathrm{R}_{\sigma}$, of the basic reproduction number $\mathrm{R}_{\mathrm{O}}$ of the deterministic special case. Whenever $\sigma>0$ then $\mathrm{R}_{\sigma}<\mathrm{R}_{\mathrm{O}}$. For small values of $\sigma$, the stability theorem guarantees almost sure exponential stability whenever $\mathrm{R}_{\sigma}<1$. We also discuss the effect of increasing $\sigma$.
\end{abstract}

\section{Introduction}

The SIR model of disease dynamics considers the population in question as being divided into three compartments, see for instance the books [1] of Anderson and May or [2] of Brauer et al. Such a model can be modified to accommodate special features of the disease, conditions in the population or interventions on the epidemic. A combination of such interventions has been studied in the paper [3] of Meng and Chen, for instance. SIR models efficiently handle cases for which the disease takes effect relatively quickly in a newly infected individual. In cases where there are significant periods of incubation of the pathogen in a newly found host, it becomes necessary to accommodate this period of incubation when modeling, possibly by means of a delay or by introduction of another class (usually denoted by $E$ for exposed) to capture newly infected individuals in the latent or incubation phase. SEIR models have been studied very extensively. Tuberculosis and HIV are examples of infections that are commonly modeled by including such a latent class. Among others, the paper [4] of Guo and Li presents a model which provides for several levels of intensity of infection. There are also models with multiple stages for other diseases, such as for instance the HIV model in Ref. [5] of Bhunu et al.

Another addition in the modeling of population dynamics of diseases is the introduction of stochasticity into models. This can bring to light new insights. Many systems in nature reveal stochasticity in itself or are subjected to stochastic perturbations. The need may arise from time to time, to accommodate such random phenomena into the modeling. Various approaches have been taken towards 
incorporating random phenomena of nature into epidemiological models. One such approach is to utilize stochastic differential equations, and in the paper [6] of Gray et al. such models are referred to as sde models. Also, many different disease types have been modeled using sde's. Modeling of HIV through sde's appears, for instance, in the papers [7] by Dalal et al., [8] by Ding et al., [9] by Yang et al. and [10] by Mukandavire et al. The papers [11] by Tornatore et al. and [12] of Jovanović and Krstić present sde models of vector-borne diseases.

An important aspect that is investigated for a disease is the long term prevalence of the disease or whether it will ultimately vanish from the population. In fact, these stability studies may well be the most active research theme in epidemiological modeling. There are various notions of stability for systems of sde's, see the book [13] of Mao. Stochastic perturbation is capable of improving stability, or to stabilize an otherwise unstable equilibrium solution of a system. This point is discussed in Ref. [13] for instance. It is also illustrated in the paper [6] for the case of disease-free equilibrium of an SIS model and in Ref. [14].

In this paper we study an sde model with independent perturbations for a disease of the SEIR type. This means that starting with a certain deterministic compartmental model, the differential equations are perturbed by mutually independent white noise terms. In particular, for this type of model, the total population size itself is perturbed directly by white noise. Examples of such models can be found in the works [15,16,10,17-19] for instance. An sde model of an SEIR disease is studied in the paper [17] of Yang et al. The papers [19] of Yuan et al. and [18] of Yang and Mao study stability of sde multi-group SEIR models. The model used in this paper can be obtained as special cases of models in Refs. $[17,18]$. We prove a theorem on almost sure exponential stability, which shows that the stochastic perturbation enhances stability of the disease-free equilibrium. Sample simulations are presented to illustrate the main theorem.

\section{Preliminaries}

We assume throughout the paper that we have a filtered probability space

$$
\left(\Omega, \widetilde{F},\left\{\widetilde{F}_{t}\right\}_{t \geq t_{0}}, \mathbb{P}\right) \text {, which is complete and right continuous. }
$$

Let us consider a $k$-dimensional Wiener process $B(t)$ defined on this filtered probability space. Suppose that we have a $k$-dimensional stochastic differential equation with initial value as below.

$$
\mathrm{d} x(t)=f(x(t), t) \mathrm{d} t+g(x(t), t) \mathrm{d} B(t), \quad \text { with } x(0)=x_{0} .
$$


The solution is denoted by $x\left(t, x_{0}\right)$. Let us suppose that $f(t, 0)=g(t, 0)=0$ for all $t \geq$ $\mathrm{O}$, so that the zero of $\mathrm{R}^{k}$ is an equilibrium point of Eq. (2.1).

There are different variations of the notion of stability for equilibrium points of sde's. We shall focus on one of them.

Definition 2.1 ([13]). The equilibrium $x=0$ of the system (2.1) is said to be almost surely exponentially stable on a subset $\Delta$ of $\mathrm{R}^{k}$ if for all $x_{\mathrm{O}} \in \Delta$,

$$
\limsup _{t \rightarrow \infty} \frac{1}{t} \ln \left|x\left(t, x_{0}\right)\right|<0 \text {. }
$$

The differential for a function $V(t, x) \in C^{1,2}\left(\mathrm{R} \times \mathrm{R}^{k}\right)$ by the formula

$$
\mathcal{L} V=\frac{\partial V}{\partial t}+f^{\operatorname{tr}} \frac{\partial V}{\partial x}+\frac{1}{2} \operatorname{Trc}\left[g^{\operatorname{trp}} \frac{\partial^{2} V}{\partial x^{2}} g\right]
$$

where Trc means trace and trp denotes the transpose of a matrix. We require the following elementary inequality in the proof of the main result. Lemma 2.2. For any real numbers $a_{1}, a_{2}, \ldots, a_{k}$ we have:

$\sum_{r=1}^{k} a_{r}^{2} \geq \frac{1}{k}\left(\sum_{r=1}^{k} a_{r}\right)^{2}$

Proof. Let $a=\sum_{r=1}^{k} a_{r}$. The statement is clearly true when $a=0$. Otherwise we solve the constrained optimization problem:

$$
\text { Minimize } \sum_{r=1}^{k} x_{r}^{2} \text { subject to } \sum_{r=1}^{k} x_{r}=a \text {. }
$$

The lemma follows readily.

The following lemma on sequences of positive real numbers is quoted from Ref. [14] where it was proved and applied. It fulfils a similar role in this paper.

Lemma 2.3 $\left(\left[14\right.\right.$, Lemma 2.2]). For $k \in \mathrm{N}$, let $X(t)=\left(X_{1}(t), X_{2}(t), \ldots, X_{k}(t)\right)$ be a bounded $\mathrm{R}^{k}$-valued function. Let $\left(t_{\mathrm{O}, n}\right)$ be any increasing unbounded sequence of positive real numbers. Then there is a family of sequences $(t l, n)$ such that for each $l \in$ $\{1,2, \ldots, k\},(t l, n)$ is a subsequence of $(t-1, n)$ and the sequence $X l(t l, n)$ converges to the largest limit point of the sequence $X l(t l-1, n)$. 


\section{The SEIR model}

When introducing stochastic perturbations into a compartmental model, one may wish to perturb a specific parameter, or some or all of the variables. So for example the model of Ref. [7, p. 39] has only two of the four state variables carrying stochastic perturbations while in the model of Ref. [17] every variable is perturbed. There are models in which the transmission rate from susceptible to infected is perturbed such as in the Refs. $[6,16,20]$ and others. We propose a model in which stochasticity is directly imposed on all the classes other than the class of susceptibles. This could be motivated by the uncertainty and behavioral change of individuals subsequent to infection. The model we present is a special case of models in Refs. [17,18].

The symbols $S$ (susceptible), $E$ (exposed), $I$ (infectious) and $R$ (removed) denote the number of individuals in the different compartments. The total population size at time $t$ is $N(t)$. Therefore $N(t)=S(t)+E(t)+I(t)+R(t)$. The mortality rates for the different classes are $\mu_{\mathrm{O}}, \mu_{1}, \mu_{2}$ and $\mu_{3}$. The $S$-class experiences an inflow at a constant rate $\mu_{\mathrm{O}} K$ . The effective rate of contact between infectives and susceptibles is $\beta$. The symbol $u$ denotes the transmission rate from the latent class to the class of infectives and $v$ is the recovery rate. The model parameters are all assumed to be positive constants. The dynamics of the disease in the population is given by the following system of sde's. The symbol $W(t)=\left(W_{1}(t), W_{2}(t), W_{3}(t)\right)$ denotes a 3 -dimensional Wiener process. The nonnegative constants $\sigma_{1}, \sigma_{2}$ and $\sigma_{3}$ denote the intensities of the stochastic perturbations.

\subsection{The sde system}

$$
\begin{aligned}
& \begin{aligned}
\mathrm{d} S & =\left[\mu_{0}(K-S)-\beta S I\right] \mathrm{d} t \\
\mathrm{~d} E & =\left[\beta S I-\left(u+\mu_{1}\right) E\right] \mathrm{d} t+\sqrt{6} \sigma_{1} E \mathrm{~d} W_{1} \\
\mathrm{~d} I & =\left[u E-\left(v+\mu_{2}\right) I\right] \mathrm{d} t+\sqrt{6} \sigma_{2} I \mathrm{~d} W_{2} \\
\mathrm{~d} R & =\left[v I-\mu_{3} R\right] \mathrm{d} t+\sqrt{6} \sigma_{3} R \mathrm{~d} W_{3} .
\end{aligned} \\
& \text { We assume that throughout the paper that } \\
& \mu_{0} \leq \min \left\{\mu_{1}, \mu_{2}, \mu_{3}\right\} . \\
& \text { Let us write } \\
& \sigma=\min \left\{\sigma_{1}, \sigma_{2}, \sigma_{3}\right\} .
\end{aligned}
$$

\subsection{An analogue of the basic reproduction number}

The following invariant $\mathrm{R}(\sigma,-)$ fulfils a central role in the main theorem on stability, similar to the role of the basic reproduction number in a deterministic $\mathrm{m}$ nt to introduce a new parameter, a constant $c$, which is not a model parameter. Thus, for $0<c \leq 1$, the number is defined as:

$$
\mathcal{R}_{(\sigma, c)}=\frac{u \beta K}{\left(\mu_{1}+u+c \sigma^{2}\right)\left(\mu_{2}+v+c \sigma^{2}\right)} .
$$


We note that in the deterministic case of the model system (3.1), i.e., when $\sigma_{1}=\sigma_{2}=$ $\sigma_{3}=0$, then the aforementioned invariant coincides with the basic reproduction number $\mathrm{R}_{O}$ of the disease, as calculated in Ref. [4]. We shall write $\mathrm{R}_{(\sigma, 1)}$ simply as $\mathrm{R} \sigma$.

\subsection{Positivity of solutions}

In order for our model to be realistic, solutions will have to be non-negative. In our quest for feasible solutions we shall refer to the set $\Delta$, which is defined as follows.

$$
\Delta=\left\{x \in \mathbb{R}^{4} \mid x_{1}>0, x_{2}>0, x_{3}>0, x_{4}>0\right\}
$$

The existence of positive solutions can be deduced from Ref. [17, Theorem 3.1] or Ref. [18, Theorem 3.1]. Thus, for each $\left(S_{\mathrm{O}}, E_{\mathrm{O}}, I_{\mathrm{O}}, R_{\mathrm{O}}\right) \in \Delta$, sde system 3.1 admits a unique solution ( $S(t), E(t), I(t), R(t))$ on $t \geq 0$, and this solution remains in $\Delta$ almost surely.

In the models of Refs. $[17,18]$, the non-zero values for the diffusion parameters associated with the $S$-classes, complicate the convergence possibilities. Since in our model we have no perturbation to $S$, convergence of $S$ becomes much more tractable. The following subset $\Omega_{1}$ of sample paths will be of interest.

$$
\Omega_{1}=\{w \in \Omega \mid S(t, w(t)), E(t, w(t)), I(t, w(t)), R(t, w(t)) \in \Delta \text { for } t \geq 0\} .
$$

Ref. [18] it follows that $\mathrm{P}\left(\Omega \backslash \Omega_{1}\right)=\mathrm{o}$. In the remainder of this paper we assume that sample paths are restricted to $\Omega_{1}$.

Proposition 3.4. If $\left(S_{\mathrm{O}}, E_{\mathrm{O}}, I_{\mathrm{O}}, R_{\mathrm{O}}\right) \in \Delta$, then almost surely, $S(t) \leq K$ for all $t>0$.

Proof. Given any path (in $\Omega_{1}$ ), then

$$
\frac{\mathrm{d}(S-K)}{\mathrm{d} t}=-\mu_{0}(S-K)-\beta S I \leq-\mu_{0}(S-K)
$$

Therefore $S(\mathrm{o})<K$ implies that $S(t)<K$ for all $t>0$.

In the following remarks we present some notation and observations that are important for the proof of the main theorem. 
Remark 3.5. Let us fix any positive real numbers $a_{0}, a_{1}, a_{2}, a_{3}$. We define the following stochastic processes:

$$
\begin{aligned}
& z(X(t))=a_{0}(K-S(t))+a_{1} E(t)+a_{2} I(t)+a_{3} R(t), \\
& Y_{E}(X(t))=\frac{E(t)}{z(X(t))}
\end{aligned}
$$

and similarly

$$
Y_{I}=z^{-1} I, \quad Y_{R}=z^{-1} R, \quad Y_{Q}=z^{-1}(K-S) .
$$

Note that for every $t>0$ we have:

$$
0<Y_{E}(t) \leq \frac{1}{a_{1}}, \quad 0<Y_{I}(t) \leq \frac{1}{a_{2}}, \quad 0<Y_{R}(t) \leq \frac{1}{a_{3}}, \quad 0<Y_{Q}(t) \leq \frac{1}{a_{0}} .
$$

In particular, the stochastic processes are bounded above by $\max \left\{\frac{1}{a_{0}}, \frac{1}{a_{1}}, \frac{1}{a_{2}}, \frac{1}{a_{3}}\right\}$, and are non-negative. Also we have the following identity:

$$
\text { for every } t>0, \quad a_{0} Y_{Q}(t)+a_{1} Y_{E}(t)+a_{2} Y_{I}(t)+a_{3} Y_{R}(t)=1 .
$$

Remark 3.6. The following stochastic processes will be encountered in the proof of the main theorem. Let

$$
M(t)=\sqrt{6} \sum_{l=1}^{3} M_{l}(t)
$$

with

$$
M_{1}(t)=\int_{0}^{t} \frac{\sigma_{1} E(y)}{z(X(y))} \mathrm{d} W_{1}(y), \quad M_{2}(t)=\int_{0}^{t} \frac{\sigma_{2} I(y)}{z(X(y))} \mathrm{d} W_{2}(y), \quad M_{3}(t)=\int_{0}^{t} \frac{\sigma_{3} R(y)}{z(X(y))} \mathrm{d} W_{3}(y) .
$$

Regarding the quadratic variations of the stochastic integral $M_{l}(t)$ we have

$$
\int_{0}^{t}\left(\frac{\sigma_{1} E(y)}{z(X(y))}\right)^{2} \mathrm{~d} y \leq \frac{\sigma_{1}^{2} t}{a_{1}^{2}}, \quad \int_{0}^{t}\left(\frac{\sigma_{2} I(y)}{z(X(y))}\right)^{2} \mathrm{~d} y \leq \frac{\sigma_{2}^{2} t}{a_{2}^{2}}, \quad \int_{0}^{t}\left(\frac{\sigma_{3} R(y)}{z(X(y))}\right)^{2} \mathrm{~d} y \leq \frac{\sigma_{3}^{2} t}{a_{3}^{2}} .
$$

We now apply the strong law of large numbers for local martingales, as in Ref. [13, p. 12] for instance. We can deduce that

$$
\lim _{t \rightarrow \infty} \frac{1}{t} \sum_{l=1}^{3} M_{l}(t)=0 \quad \text { (a.s.). }
$$

\section{Stability theorems}

Stability results of sde models for bigger values as well as smaller values of the perturbation parameters appear, for instance, in Ref. [20, Theorem 5], Ref. [7, p. 52] and in Ref. [14]. In the main theorem, Theorem 4.1, we investigate stability for mainly the smaller values of the perturbation parameters. Recall that $\sigma=\min \left\{\sigma_{j} \mid j=1,2,3\right\}$. 
Theorem 4.1. Suppose that $c$ is a constant with $\mathrm{o}<c \leq 1$ and such that the following inequality holds:

$c \sigma^{2} \leq \mu_{0}$.

If $\mathrm{R}_{\sigma, c}<1$, then the disease-free equilibrium is almost surely exponentially stable.

Proof. Let us assume the conditions of the theorem to hold. The condition $\mathrm{R}_{\sigma} c<1$ is equivalent to the following inequality:

$$
\frac{u}{\mu_{1}+u+c \sigma^{2}} \beta K-\left(\mu_{2}+v+c \sigma^{2}\right)<0 .
$$

We can choose a number $a_{0}>0$ sufficiently small in order that the following inequality holds:

$$
\left(a_{0}+\frac{u+a_{0}}{\mu_{1}+u+c \sigma^{2}}\right) \beta K-\left(\mu_{2}+v+c \sigma^{2}\right)<0 .
$$

Now we fix the following numbers. Let

$$
a_{1}=\frac{u+a_{0}}{\mu_{1}+u+c \sigma^{2}} \text { and let } a_{2}=1 \text {. }
$$

We can choose a positive number a3 sufficiently small, such as to satisfy the inequality:

$$
\left(a_{0}+\frac{u+a_{0}}{\mu_{1}+u+c \sigma^{2}}\right) \beta K-\left(\mu_{2}+v+c \sigma^{2}\right)+a_{3} v<0 .
$$

Let us write ( $S(t), E(t), I(t), R(t))=X(t)$. With the numbers $a_{i}$ as declared above, we define a stochastic process $z(X(t))$ as in remark 3.5. Since $z(X(t))>0$ for all $t>0$ (recall that we assume the sample paths to be in $\Omega_{1}$ ), we can also define $X(t)$ ).

$z(X(t))=a_{\mathrm{O}}(K-S(t))+a_{1} E(t)+a_{2} I(t)+a_{3} R(t), V(X(t))=\ln z(X(t))$.

In order to prove our theorem it suffices to prove that $z(X(t))$ converges exponentially to zero (a.s.). Using Itô's formula (see Ref. [13] for instance) we can express the stochastic process $V(X(t))$ as: 


$$
V(X(t))=V(X(0))+\int_{0}^{t} \mathcal{L} V(X(u)) \mathrm{d} u+M(t)
$$

with $M(t)$ as in Remark 3.6, where we noted that

$$
\lim _{t \rightarrow \infty} \frac{1}{t} M(t)=0 \quad \text { (a.s.). }
$$

Therefore

$$
\left.\limsup _{t \rightarrow \infty} \frac{1}{t} V(X(t))=\limsup _{t \rightarrow \infty} \frac{1}{t} \int_{0}^{t} \mathcal{L} V(X(y)) \mathrm{d} y \quad \text { (a.s. }\right)
$$

and it follows that we only need to prove that

$$
\limsup _{t \rightarrow \infty} \mathcal{L} V(X(t))<0 \quad \text { a.s. }
$$

We write down the expression for $\operatorname{LV}(\mathrm{X}(\mathrm{t}))$, given the system of stochastic differential equations of the SEIR model.

$$
\begin{aligned}
\mathcal{L} V(X)= & \frac{1}{z}\left[-a_{0}\left(\mu_{0}(K-S)-\beta S I\right)+a_{1}\left(\beta S I-\left(u+\mu_{1}\right) E\right)+a_{2}\left(u E-\left(v+\mu_{2}\right) I\right)+a_{3}\left(v I-\mu_{3} R\right)\right] \\
& -\frac{3}{z^{2}}\left(\left(a_{1} \sigma_{1} E\right)^{2}+\left(a_{2} \sigma_{2} I\right)^{2}+\left(a_{3} \sigma_{3} R\right)^{2}\right) .
\end{aligned}
$$

For every sample path $\mathrm{w}$ of the 3-dimensional Wiener process $\mathrm{W}(\mathrm{t})$, there exists an unbounded increasing sequence $\left(\tau_{n}^{w}\right)$ of positive time values for which

$$
\lim _{n \rightarrow \infty} \mathcal{L} V\left(X\left(\tau_{n}, w\left(\tau_{n}\right)\right)\right)=\limsup _{t \rightarrow \infty} \mathcal{L} V(X(t, w(t)))
$$

Fix such a sequence. Then by Lemma 2.3 there exists a subsequence $\left(\tau_{n}^{w}\right)$ for which the following limit exists (see notation in Remark 3.6):

$$
\lim _{n \rightarrow \infty}\left(S\left(t_{n}^{w}, w\left(t_{n}^{w}\right)\right), Y_{E}\left(X\left(t_{n}^{w}, w\left(t_{n}^{w}\right)\right)\right), Y_{I}\left(X\left(t_{n}^{w}, w\left(t_{n}^{w}\right)\right)\right), Y_{R}\left(X\left(t_{n}^{w}, w\left(t_{n}^{w}\right)\right)\right)\right)
$$


This enables us to define the following limits (and now we suppress the path $\mathrm{w}$ in the notation):

$$
\begin{aligned}
& s=\lim _{n \rightarrow \infty} S\left(t_{n}\right), \quad f=\lim _{n \rightarrow \infty} Y_{E}\left(X\left(t_{n}\right)\right), \quad i=\lim _{n \rightarrow \infty} Y_{I}\left(X\left(t_{n}\right)\right), \quad r=\lim _{n \rightarrow \infty} Y_{R}\left(X\left(t_{n}\right)\right), \\
& q=\lim _{n \rightarrow \infty} Y_{Q}\left(X\left(t_{n}\right)\right) .
\end{aligned}
$$

From the identity (3.2) it follows that

$a_{0} \quad q+a_{1} f+a_{2} i+a_{3} r=1$.

Let us write

$$
\Lambda=\lim _{n \rightarrow \infty} \mathcal{L}\left(\ln \left[z\left(t_{n}\right)\right]\right) .
$$

Then from the Eq. (4.3) it follows that

$$
\begin{aligned}
\Lambda= & -a_{0} \mu_{0} q+a_{0} \beta s i+a_{1}\left(\beta s i-\left(u+\mu_{1}\right) f\right)+a_{2}\left(u f-\left(v+\mu_{2}\right) i\right) \\
& +a_{3}\left(v i-\mu_{3} r\right)-3\left(\left(a_{1} \sigma_{1} f\right)^{2}+\left(a_{2} \sigma_{2} i\right)^{2}+\left(a_{3} \sigma_{3} r\right)^{2}\right) .
\end{aligned}
$$

In the sequence of inequalities which follows below, we use the fact that $c \leq 1$ implies $-\sigma^{2} \leq-(c \sigma)^{2}$. At some point we e identity $a_{1} f+a_{2} i+a_{3} r=1-a_{\mathrm{O}} q$ and the inequality $a_{1} f+a_{2} i+a_{3} r \leq 1$, both of which follows from the identity (4.4).

$$
\begin{aligned}
-\left(a_{1} \sigma_{1} f\right)^{2}-\left(a_{2} \sigma_{2} i\right)^{2}-\left(a_{3} \sigma_{3} r\right)^{2} & \leq-c \sigma^{2}\left[\left(a_{1} f\right)^{2}+\left(a_{2} i\right)^{2}+\left(a_{3} r\right)^{2}\right] \\
& \leq-\frac{1}{3} c \sigma^{2}\left(a_{1} f+a_{2} i+a_{3} r\right)^{2} \quad(\text { from Lemma 2.2) } \\
& =-\frac{c \sigma^{2}}{3}\left(a_{1} f+a_{2} i+a_{3} r\right)\left(1-a_{0} q\right) \\
& =-\frac{c \sigma^{2}}{3}\left(a_{1} f+a_{2} i+a_{3} r\right)+\frac{c \sigma^{2}}{3}\left(a_{1} f+a_{2} i+a_{3} r\right) a_{0} q \\
& \leq-\frac{c \sigma^{2}}{3}\left(a_{1} f+a_{2} i+a_{3} r\right)+\frac{c \sigma^{2}}{3} a_{0} q \\
& \leq-\frac{c \sigma^{2}}{3}\left(a_{1} f+a_{2} i\right)+\frac{c \sigma^{2}}{3} a_{0} q .
\end{aligned}
$$

Therefore we obtain the following inequality:

$\Lambda \leq-a_{\mathrm{O}} \mu_{\mathrm{O}} q+a_{\mathrm{O}} \beta s i+a_{1}\left(\beta s i-\left(u+\mu_{1}\right) f\right)+a_{2}\left(u f-\left(v+\mu_{2}\right) i\right)$

$+a_{3}\left(v i-\mu_{3} r\right)-c \sigma^{2}\left(a_{1} f+a_{2} i\right)+c \sigma^{2} a_{0} q$.

From Proposition 3.4 it follows that $s \leq K$. Furthermore we observe that 
$-a_{1}\left(u+\mu_{1}+c \sigma^{2}\right) f+a_{2} u f=-a_{0} f$.

This yields

$\Lambda \leq i\left[\left(a_{\mathrm{O}}+a_{1}\right) \beta K-\left(v+\mu_{2}\right)+a_{3} v\right]-a_{\mathrm{O}} \mu_{\mathrm{O}} q-a_{\mathrm{O}} f-a_{3} \mu_{3} r-c \sigma^{2} a_{2} i+c \sigma^{2} a_{\mathrm{O}} q$.

Therefore we obtain an inequality

$\Lambda \leq A_{\mathrm{O}} q+A_{1} f+A_{2} i+A_{3} r$

with

$$
\begin{aligned}
& A_{0}=a_{0}\left[c \sigma^{2}-\mu_{0}\right], \\
& A_{1}=-a_{0}, \\
& A_{2}=\left(a_{0}+\frac{u+a_{0}}{\mu_{1}+u+c \sigma^{2}}\right) \beta K-\left(\mu_{2}+v+c \sigma^{2}\right)+a_{3} v, \\
& A_{3}=-a_{3} \mu_{3} .
\end{aligned}
$$

From the inequality (4.2) it follows that $A_{2}<0$. The coefficient $A_{\mathrm{O}}$ is negative due to the condition (4.1). Therefore all the coefficients $A_{\mathrm{O}}, A_{1}, A_{2}$ and $A_{3}$ are negative. Furthermore, $a_{0} i+a_{1} q+a_{2} r+a_{3} f=1$ and thus the limits $i, q, r, f$ cannot all be zero. Therefore $\Lambda<0$, and the proof is complete.

Remark 4.2. The role of the constant $c$ in Theorem 4.1 is as follows. If the requirements of Theorem 4.1 are fulfilled for a given value $\sigma^{*}$ of $\sigma$, then by increasing the value of $\sigma$ we will still have the disease-free equilibrium to be almost surely exponentially stable.

Theorem 4.3. If $\mathrm{R}_{\mathrm{O}}<1$ then the model is almost surely exponentially stable. 


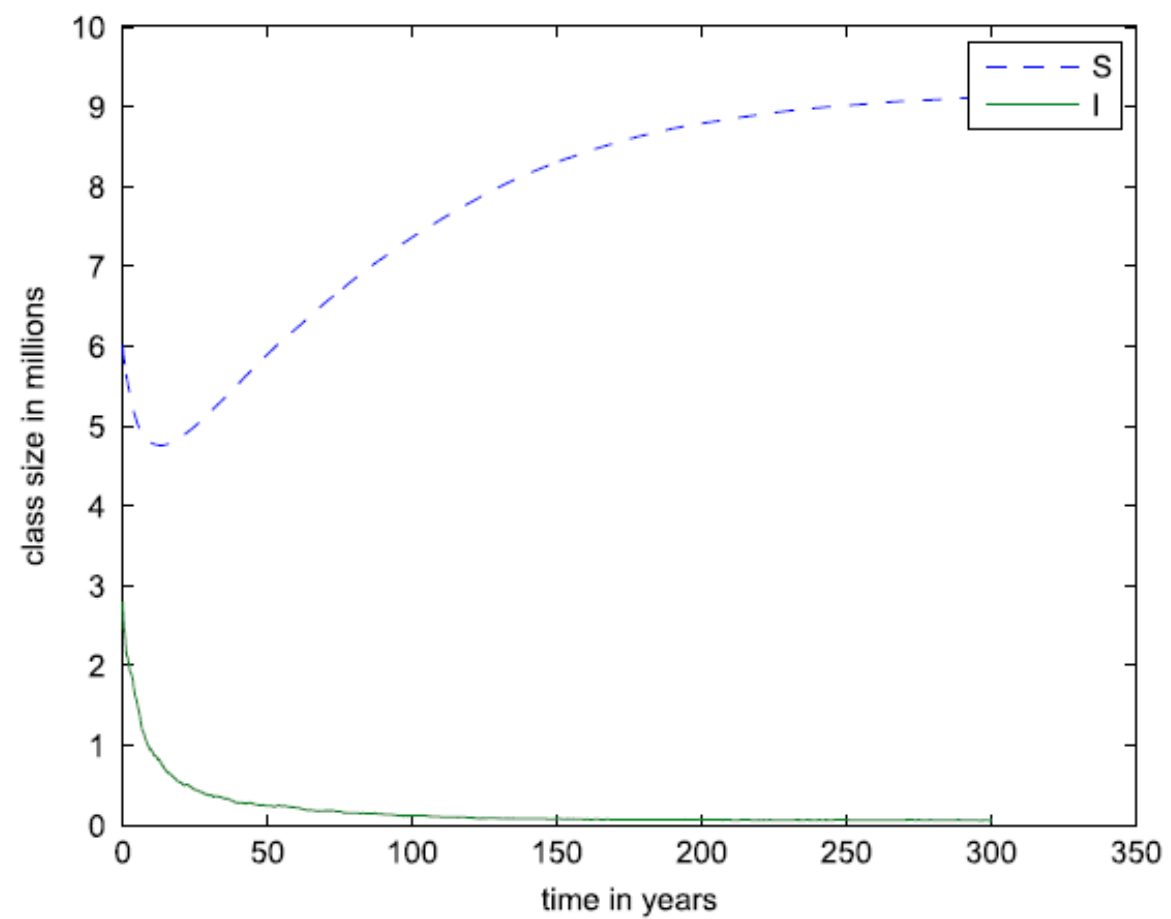

Fig. 1. For $\mathcal{R}_{(\sigma, 1)}=1.0510>1$, Theorem 4.1 does not guarantee almost sure exponential stability.

Proof. The proof follows very much along the argument of the proof of Theorem 4.1, but is much simpler in that we completely ignore the diffusion parameters. We choose $a_{1}=\left(u+a_{0}\right)\left(\mu_{1}+u\right)^{-1}$ and the Eq. (4.5) is simplified to get the inequality:

$\Lambda \leq-a_{0} \mu_{0} q+a_{0} \beta s i+a_{1}\left(\beta s i-\left(u+\mu_{1}\right) f\right)+a_{2}\left(u f-\left(v+\mu_{2}\right) i\right)+a_{3}\left(v i-\mu_{3} r\right)$.

The proof follows similarly as for Theorem 4.1.

\section{Numerical examples}

The graphs in Figs. 1-3 were simulated with the model parameters as follows:

$K=10$ million $, \quad \beta=0.024, \quad u=0.05, \quad v=0.15$,

$\mu_{\mathrm{O}}=0.017, \quad \mu_{1}=0.0172, \quad \mu_{2}=0.0185, \quad \mu_{3}=0.0172$,

and initial conditions (in units of millions)

$S_{O}=6, \quad E_{O}=1, \quad I_{O}=2.8, \quad R_{O}=0.2$.

The simulations run over different time horizons as indicated on the graphs. Now we note that these parameters give in the deterministic case (i.e., with $\sigma=0$ ), a value of $\mathrm{R}_{0}$ $=1.0598$ to the basic reproduction number. Consequently the system eventually approaches an endemic equilibrium point with coordinates: 


$$
\begin{aligned}
S^{*} & =\frac{u v+u \mu_{2}+v \mu_{1}+\mu_{1} \mu_{2}}{u \beta}, \\
E^{*} & =\frac{\mu_{0}\left(u K \beta-u v-u \mu_{2}-v \mu_{1}-\mu_{1} \mu_{2}\right)}{u \beta\left(u+\mu_{1}\right)}, \\
I^{*} & =\frac{\mu_{0}\left(u K \beta-u v-u \mu_{2}-v \mu_{1}-\mu_{1} \mu_{2}\right)}{\beta\left(u v+u \mu_{2}+v \mu_{1}+\mu_{1} \mu_{2}\right)}, \\
R^{*} & =\frac{v \mu_{0}\left(u K \beta-u v-u \mu_{2}-v \mu_{1}-\mu_{1} \mu_{2}\right)}{\mu_{3} \beta\left(u v+u \mu_{2}+v \mu_{1}+\mu_{1} \mu_{2}\right)} .
\end{aligned}
$$

The numerical values are:

$S^{*}=9.4360, \quad E^{*}=0.1427, \quad I^{*}=0.0423, \quad R^{*}=0.3692$.

The graphs show only the curves $S$ and $I$, and we note (from the system of sde's) that $S^{\prime}(t)$ is continuous.

For the simulation of Fig. 1, we choose $\sigma=0.02$. Then we obtain $\mathrm{R}(\sigma, 1)=1.0510$. A different value of $c$ will not decrease the value of $\mathrm{R}(\sigma, c)$. Therefore Theorem 4.1 does not guarantee almost sure exponential stability in this case.

In Fig. 2 we use $\sigma=0.1$ and then we have $\mathrm{R}(\sigma, 1)=0.8708<1$. With the requirements of Theorem 4.1 fulfilled, in this case the theorem asserts that the disease-free equilibrium is almost surely exponentially stable. 


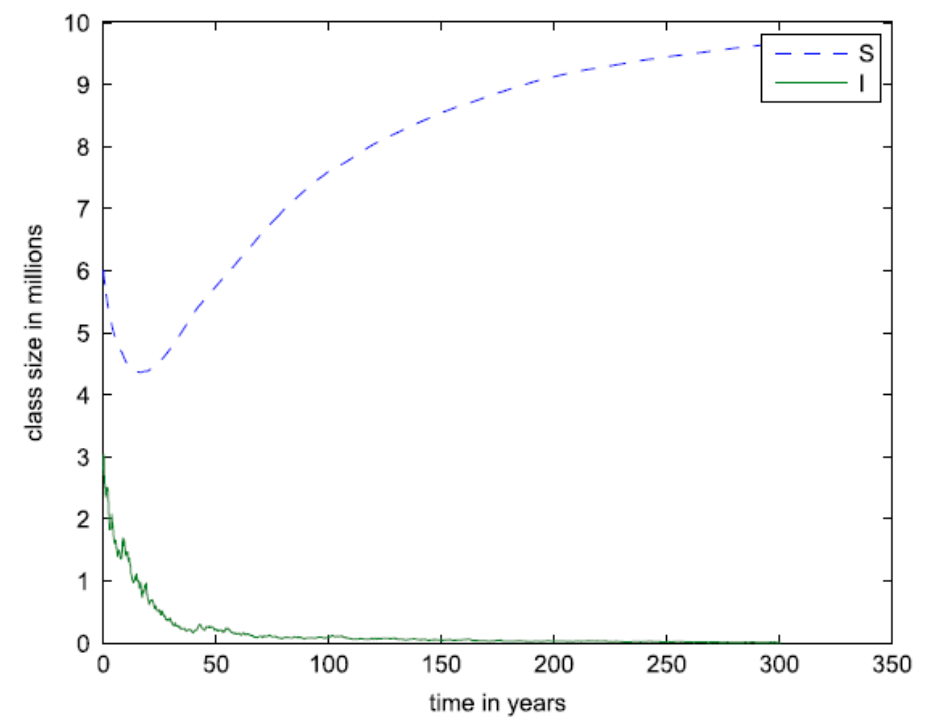

Fig. 2. Here we have $\mathcal{R}_{(\sigma, 1)}=0.8708<1$, and by Theorem 4.1 the disease-free equilibrium is almost surely exponentially stable.

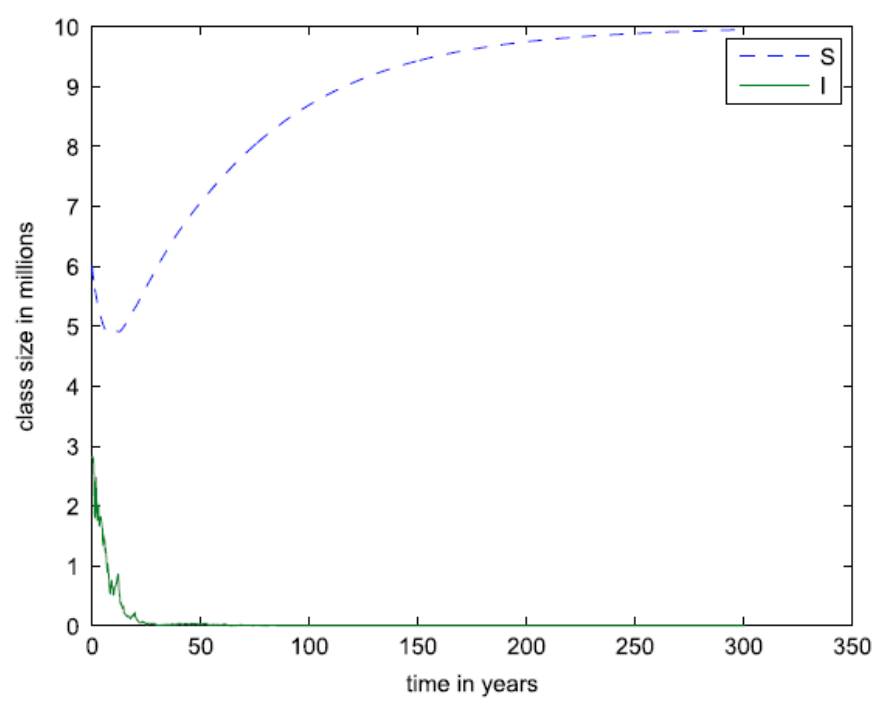

Fig. 3. With $\sigma=0.25$, bigger than in Fig. 2, and the other parameters the same, we expect a more rapid convergence to the disease-free.

Having fulfilled the conditions of Theorem 4.1 with our previous choice of parameters and led by Remark 4.2, we choose $\sigma=0.25$, and we expect the disease-free equilibrium to have almost sure exponential stability. In fact in this case, convergence to the disease-free equilibrium is expected to happen faster. Fig. 3 shows a simulation of the solution paths.

Finally, we show that the condition $\mathrm{R}(\sigma, c)<1$ is not sufficient to guarantee almost sure exponential stability of the disease- free equilibrium. In Fig. 4 we show a simulation with all the deterministic parameter values as before except that $\beta$ is chosen at a higher value; $\beta=0.04$. Taking $\sigma=0.25$ gives $\mathrm{R}(\sigma, 1)=0.6675<1$ (deterministic, $\mathrm{R}_{0}=1.7662$ ). For all the model parameters as chosen now, it is impossible to find a positive number $c$ to fulfil the condition $c \sigma^{2} \leq \mu_{\mathrm{O}}$ of Theorem 4.1. Consequently the theorem does not guarantee the disease-free 
equilibrium to be almost surely exponentially stable. Indeed, the graph in Fig. 4 does not show any tendency towards the disease-free equilibrium.

\section{Conclusion}

There are only a few research publications on sde modeling of SEIR dynamics. The paper [18] presents results on long time behavior of the system and in particular on convergence of $S$ to its disease-free equilibrium value. It includes a study of the asymptotic probability distribution of the variables $S k$. In the paper [17] is proved a theorem on the asymptotic value of the time average of the deviation from the disease-free equilibrium, subject to certain upper bounds on the perturbation parameters and with $\mathrm{R}_{O}$ assumed to not exceed unity. The same paper gives results on limits of time averages, in particular, $m$ value. Also, for the special case of $S$ being unperturbed (as in our model), in Ref. [17] the system is shown to be stochastically stable in the large, see Ref. [17, Remark 3.1].

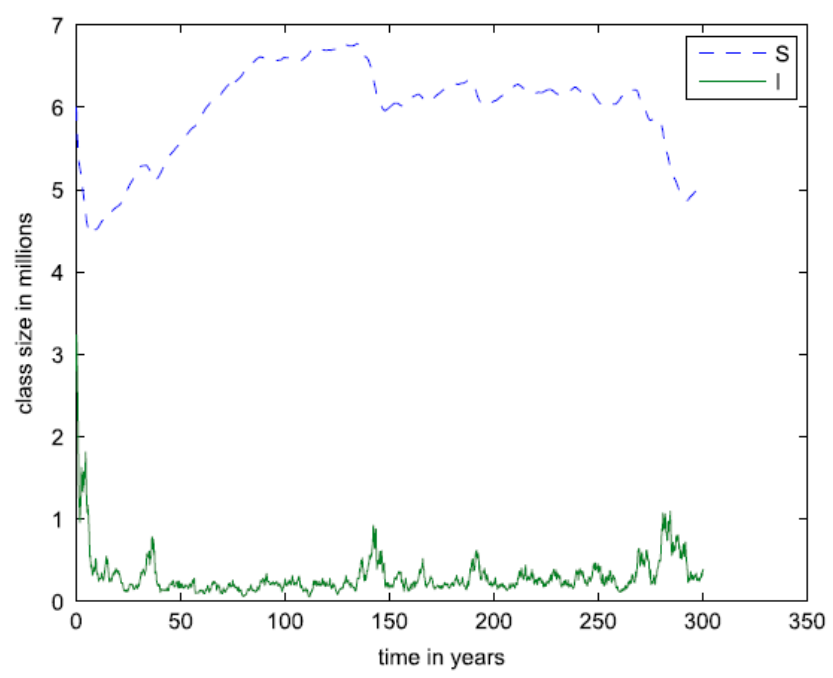

Fig. 4. In Theorem 4.1, the condition $\mathcal{R}_{(\sigma, c)}<1$ is not sufficient to guarantee almost sure exponential stability of the disease-free equilibrium.

In relation to the theme of this paper, we particularly want to note the paper [6] which proves a stability result allowing for $R_{O}$ to exceed the value 1 . The current paper makes a useful contribution to understanding the disease-free equilibrium of the given sde model of an SEIR disease. Our main result has an uncomplicated formulation and guarantees almost sure exponential stability of the disease-free equilibrium value for $\mathrm{R}_{\sigma}<1$. This implies an improvement on the condition $\mathrm{R}_{\mathrm{O}}<1$ required for local asymptotic stability in the deterministic case.

\section{Acknowledgement}

The author acknowledges funding from the National Research Foundation. 


\section{References}

[1] R.M. Anderson, R.M. May, Infectious Diseases of Humans: Dynamics and Control, Oxford University Press, London, New York, 1991.

[2] Fred Brauer, Pauline van den Driessche, Jianhong Wu, Mathematical Epidemiology, in: Lecture Notes in Math., vol. 1945, Springer, Berlin, 2008.

[3] Xinzhu Meng, Lansun Chen, The dynamics of a new SIR epidemic model concerning pulse vaccination strategy, Applied Mathematics and Computation 197 (2) (2008) 582597.

[4] H. Guo, Michael Y. Li, Global dynamics of a staged progression model for infectious diseases, Mathematical Biosciences and Engineering 3 (3) (2006) 513-525.

[5] C.P. Bhunu, S. Mushayabasa, H. Kojouharov, J.M. Tchuenche, Mathematical analysis of an HIV/AIDS model: impact of educational programs and abstinence in sub-saharan Africa, Journal of Mathematical Modelling and Algorithms 10 (1) (2011) 31-55. http://dx.doi.org/10.1007/s10852-010-9134-o.

[6] A. Gray, D. Greenhalgh, L. Hu, X. Mao, J. Pan, A stochastic differential equation SIS epidemic model, SIAM Journal of Applied Mathematics 71 (3) (2011) 876-902.

[7] N. Dalal, D. Greenhalgh, X.R. Mao, A stochastic model of AIDS and condom use, Journal of Mathematical Analysis and Applications 325 (2007) 36-53.

[8] Y. Ding, M. Xu, L. Hu, Asymptotic behavior and stability of a stochastic model for AIDS transmission, Applied Mathematics and Computation 204 (2008) 99-108.

[9] J. Yang, X. Wang, X. Li, Global stability of an HIV model with stochastic perturbation, Asian-European Journal of Mathematics 4.2 (2011) 349-358.

[10] Z. Mukandavire, P. Das, C. Chiyaka, N.H. Gazi, K. Das, T. Shiri, HIV/AIDS model with delay and the effects of stochasticity, Journal of Mathematical Modelling and Algorithms 10 (2011) 181-191. http://dx.doi.org/10.1007/s10852-010-9148-7.

[11] Elisabetta Tornatore, Stefania Maria Buccellato, Parasite population delay model of malaria type with stochastic perturbation and environmental criterion for limitation of disease, Journal of Mathematical Analysis and Applications 360 (2009) 624-630.

[12] M. Jovanović, M. Krstić, Stochastically perturbed vector-borne disease models with direct transmission, Applied Mathematical Modelling 36 (2012) 5214-5228.

[13] X. Mao, Stochastic Differential Equations and Applications, Horwood, Chichester, 1997.

[14] P.J. Witbooi, Stochastic stability of an SIR epidemic model, submitted for publication.

[15] D. Jiang, C. Ji, N. Shi, J. Yu, The long time behavior of DI SIR epidemic model with stochastic perturbation, Journal of Mathematical Analysis and Applications 372 (2010) 162-180.

[16] Q. Lu, Stability of an SIRS system with random perturbations, Physica A 388 (2009) 3677-3686.

[17] Qingshan Yang, Daqing Jiang, Ningzhong Shi, Chunyan Ji, The ergodicity and extinction of stochastically perturbed SIR and SEIR epidemic models with saturated incidence, Journal of Mathematical Analysis and Applications 388 (2012) 248-271. http://dx.doi.org/10.1016/j.jmaa.2011.11.07. 
[18] Qingshan Yang, Xuerong Mao, Extinction and recurrence of multi-group SEIR epidemic models with stochastic perturbations, Nonlinear Analysis. Real World Applications 14

(3) (2013) 1434-1456. http://dx.doi.org/10.1016/j.nonrwa.2012.10.007.

[19] Chengjun Yuan, Daqing Jiang, Donal O’Regan, Ravi P. Agarwal, Stochastically asymptotically stability of the multi-group SEIR and SIR models with random perturbation, Communications in Nonlinear Science and Numerical Simulation 17 (2012) 2501-2516.

[20] A. Lahrouz, L. Omari, D. Kiouach, Global analysis of a deterministic and stochastic nonlinear SIRS epidemic model, Nonlinear Analysis. Modelling and Control 16 (2011) 59-76. 